Monatsschrift f. Geburtshülfe u. Gynäkologie 1924;66:322-326

\title{
IV. Literaturverzeichnis
}

\section{Geburtshülfe.}

Baer und Reis, Beobachtungen über Kapillarinikroskopie während der

Schwangerschaft. J. Am. med. Ass. 16. II. S. 526. Baumm, Die Diagnose des Kopfstandes unter der Geburt. Munch, med.

Woch. Nr. 10. S. 301. Biermer, Röntgenbestrahlung bei Ptyalismus gravid. Med. Klin. Nr. 8. S. 243. Burger, Fall von Status epilept. unter der Geburt und im Wochenbett.

Z. f. G. Nr. 7. S. 257. Burger, Über Hypophysenextrakt in der Geburtshilfe. Gynec. obstet.

IX. 1. S. 136. Corara und Strauß, Suprarenale Blutung bei Neugeborenen. J. Am. med.

Ass. 23. II. S. 626. Depken, Die Behandlung der Plac. praev. Z. f. G. Nr. 7 a. S. 298. Edwards, Lymphatoostomie bei Puerperalinfektion. Surg. Gyn. Obst.

Гebr. 24. S. 256. Engelmann, Die hämoklas. Krise als Leberfunktionsprüfung. Med. Klin. Nr. 10. S. 308. Fink, Schädelverletzungen durch die Kiellandzange. Z. f. G. Nr. 8. S. 501. Gal, Empfängnis bei kaum wahrnehmbarer Hymenalöffnung. Z. f. G.

Nr. 7 a. S. 338. Gerdes, Über einen Fall von Luxatio bulbi als Geburtsverletz, ung. Munch. med. Woch. Nr. 9. S. 274. Geßner, Die badische Eklampsiestatistik für das Jahr 1921 im Lichte einer

funktionellen Eklampsiebehandlung. Z. f. G. Nr. 7. S. 250. Heim, Erfahrungen mit der Kiellandzange. Z. f. G. Nr. 8. S. 506. Heinlein, Zur Therapie und Prophylaxe der Eklampsie. Z. f. G. Nr. 7 a.

S. 305. Hirst und Wachs, Geburtsbeschädigung des Steißbeins and ihre Behandlung.

Am. J. Obst. Gyn. VII. 2. Febr. 24. S. 199.

Literaturverzeiclinis.

323

Keller, Beitrag zur Genese der Mola hydatiformis. Gynec. obstetr. IX. 1.

S. 68. Koblank, Blutungen. Heb.-Ztg. Nr. 4. S. 51.

Liebermeister, Tuberkulose und Schwangerschaft. Klin. Woch. Nr. 11. S. 433. Li@gemann, Beitrag zur Gasbildung im kreißenden und puerperalen Uterus.

Z. f. G. Nr. 9. S. 529. Lönne, Über Nabelschnurzerreißung, unt·er besonderer Berücksichtigung eines seltenen Falles von isolierter Ruptur beider Umbilikalart. bei

spontaner Geburt. Z. f. G. Nr. 10/565. Milnor und Femel, Die Diagnose der Schwangerschaft. J. Amer. med. Ass.

16. II. S. 538. Neumann, Anomalien des knöehernen Scbädeldaches der Neugeborenen.

Z. f. G. Nr. 10. S. 571. Neurath, Rachitis und Nervensystem. Klin. Woch. Nr. 9. S. 337. J.

Osborn und Pollack und Phelau, Placenta accreta, Vorkommen, Pathologie

und Behandlung. Surg. Gyn. Obst. Febr. 24. S. 181. Peyser, Untersuchungen über das veget.

Nervensystem in der Schwangerschaft, Z. f. G. Nr. 8. S. 496. Ragusa, Spontane Uterusruptur in partu. La clinica ostet. Febr. 24. S. 54. Rech, Über den physiol. Verschluß der Nabelarterien. Klin. Woch. Nr. 8.

S. 323. Rosenthal, Über die Ergebnisse, die bei der Bekämpfung der Erbsyphilis 
durch stationäre Behandlung in sog. Welanderheimen bisher erzielt

worden sind. Klin. Woch. Nr. 8. S. 326. Rudolphson, Über die Frage der Sekundärnaht am Damm im Wochenbett.

Z. f. G. Nr. 7 a. S. 344. Schmilt, Über die Regulierung der plazentalen Atmung. Z. f. G. Nr. 8. S. 489. Schneidermann, Weitere Beobacht\%omgen über renale Glykosurie in der

Schwangerschaft. Am. J. Obstet. gyn. VIII. 2. Febr. 24. S. 177. Schoedel, Stillfähigkeit und Gebur† sgewichte im sächsischen Industrie-

bezirk in den Jahren 1920 und 1923. Munch, med. Woch. Nr. 9. S. 277. Schiitt, Zur Behandlung der Asphyxia pallid, neonat. Z. f. G. Nr. 10. S. 564. Schwarz, Funktionsdiagnose oder funktionelle Diagnostik? Klin. Woch.

Nr. 9. S. 365. Sharpe und Maclaire, Intrakranielle Blutung bei Neugeborenen. Surg.

Gyn. Obst. Febr. 24. S. 200. Stoeckel, Betrachtungen über die Pyelitis gravid. Munch, med.

Woch. Nr. 9.

S. 257. Streibel, Intraperitoneale Anwendung des Rivanols bei Fall von kompl.

Uterusruptur intr. part, Z. f. G. Nr. 7 a. S. 329. Vickers, Diabetes insipidus mit akuter Verhaltung während der Schwangerschaft, Surg. gyn. Obstet, Febr. 24. S. 223. Vigher, Zufälle in

Schwangerschaft und Geburt bei syphilitischen Frauen.

Paris medical 1. VIII. 24.

- $\quad$ Die Wassermannsche Reaktion in der geburtshilflichen Praxis. Les

Sciences méd. 31. I. 24.

- $\quad$ Die Medikamente, welche die Uteruskontraktionen beeinflussen. La

presse méd. 23. I. 24.

324

Literaturverzeichnis.

Gynäkologie.

Amreich, Zur Ätíologie der von den Uterusvenen ausgehenden Luftembolie.

Z. f. G. Nr. 9. S. 521. Baetzner, Die operative Behandlung der Blasentumoren. Klin. Woch.

Nr. 8. S. 325. Beck, Primäres Tubenkarzinom. Z. í. G. Nr. 10. S. 562. Berczella und Wastl, Zur

Methodik der Blutkörperchensenkungsprobe.

Munch, med. Woch. Nr. 8. S. 228. Bönníger und Herrmann, Weitere Untersuchungen über die

Blutkörperchen-

senkungsgeschwindigkeit. Klin. Woch. Nr. 10. S. 403. Bradley, Erfolge der intermittierenden

aspiratorischen Hyperämie in der

Gynäkologie. - 123 Fälle. Am. J. obst. gyn. VII. 2. Febr. 24. S. 195. Brugnatelli, Kranielle

Blutung, welche eine extrauterine Schwangerschaft

vortãuscht. La clinica obstet. Febr. 24. S. 57. Calzavara, Fall von Knochenbildung im runden Mutterband. Z. f. G.

Nr. 10. S. 579. Crowell, Schmerzen bei Erkrankungen der genii.-urinalen Wege. Surg.

Gyn. Obst. Febr. 24. S. 218. Dietrich, Die Therapie des Uterasprolapses. Klin. Woch. Nr. 10. S.

409. Eckstein, Meine Stellungnahme zum Entwurf E. Kehrers zn den Richtlinien

f. d. außerklin. operat. Technik der frühzeitigen Schwangerschafts-

unterbrechung. Z. f. G. Nr. 7 a. S. 332. Fink, Neues Material zur Bewertung der $\mathrm{W}^{1 / 8} 8^{1 / 8}$ rschen

Abortzange. Munch.

med. Woch. Nr. 10, 300. Frank, Wert diagnostischer Excochleation und Aussparung für die

Gynäkologie. J. Amer. Mod. Ass. 23. II. S. 619. Friedlcinder, Die Bhitsenkungsprobe als

diagnostisches Hilfsmittel bei 
chirurgischer Infektion. Am. J. obst. gynec. VII. 2. Febr. 24. S. 125. Frehse, Über die

strafrechtliche und ehrengerichtliche Verant $\Lambda$ vortlichkeit

des Arztes bei Todesfällen in der Narkose. Dtsch. med. Woch. Nr. 8.

S. 245. Gaifanu, Die Sonde in der Gynäkologie. La clinica ostet. Febr. 1924. S. 41. Galant, Psychogynäkologie ? Z. f. G. Nr. 7 a. S. 343. Goldschmidt, 2 Fälle von Uterusperforation mit schvverer Darnverletzung.

Dtsch. med. Woch. Nr. 8/238. Groß, Vagina dupl. mit Uterus simpl. Z. f. G. Nr. 10. S. 582.

Gumpert, Die Z\mahme erworbener Geschlechtskrankheiten bei Kindern.

Dtsch. med. Woch. Nr. 7. S. 206. Guttmacher, Diffuse uterine Drüsenzysten bei einer Katze.

Bull. Johns.

H. Hosp. Febr. 24. S. 49. Haselhorst und Peemöller, Behandlung von entzündlichen

Genitalerkran-

kungen mit der, ,verbrennungsfreien Ultrasonne”. Z. f. G. Nr. 7.

S. 237. Haupt, Untersuchungen über die Pathogenität der Trichomonas vag.

Munch, med. Woch. Nr. 7. S. 204. Hellendall, Fall von Parotitis postop. gonorrh. Z. f. G. Nr. 7.

S. 256. - Die supravaginale Amputation des Uterus bei schweren Abortblutungen

zum Zwecke rascher Bl·utstillung und gleichzeitiger Sterilisierung. Z. f. G. Nr. 7 a. S. 324.

Literaturverzeichnis.

325

Hinselmann, Über die Natur der Chaletzky-Neumannschen Zellen. Z. f. G.

Nr. 9. S. 537. Hirsch, Zum Kampf gegen die Fruchtabtreibung. Z. f. G. Nr. 9. S. 519. Hoffgaard,

Die Bedeutung der Blutkörperchensenkungsgeschwindigkeit

für die Diagnose des Karzinom. Munch, med. Woch. Nr. 8. S. 231. Jacquin, Über ein

aberrierendes Myom und seine maligne Entartung.

Myoma malignum pseudocysticum. Gynéc. obstét. IX. 1. S. 163. Jaeger, Zur Frage der

Abortbehandlung. Z. f. G. Nr. 10. S. 576. Joseph, Rivanol als Anästhetikum und Desinfiziens der Harnblase. Munch..

med. Woch. Nr. 7. S. 205. Kantor, Etwas über Kurpfuscherei. Dtsch. med. Woch. Nr. 8. S. 259.

Kaiser, Zur Zervixerweiterung nach Hegar. Z. f. G. Nr. 7 a. S. 316. Kritzler, Zur Anregung der

Darmtätigkeit nach Bauchschnitten. Dtsch.

med. Woch. Nr. 8. S. 240. -- Intrauterinpessar und Extrauteringravidität. Z. f. G. Nr. 9. S. 534.

Kuncz, Über die Heilungserfolge der Alexander-Adams-Operation. Z. f. G.

Nr. 7 a. S. 339. Li $\pi$ demann, Über die Entstehung von Verbrennungen bei Diathermie.

Z. f. G. Nr. 7 a, S. 318. Madlener, Die Forderung der Errichtung aseptischer Abteiiuneen in

Krankenhäusern. Z. f. G. Nr. 7. S. 259. Mays, C, und Walters, Transplantation des Ureters in das

Rektum. Journ.

Amer. med. Ass. Febr. 23. II. S. 624. Mailer, Über eine neue Therapie des weiblichen Fluors.

Med. Klin. Nr. 7.

S. 216. Penkert, Mastdarm-Gebärmutter-Bauchdeckenfistel nach tuberkulöser Ei-

leitererkrankung. Z. f. G. Nr. 7. S. 247. - Seltene Spätrezidive nach Karzinomoperation. Z. f. G.

Nr. 9. S. 538. Pfeilsticker, Temporäre Tubensterilisation. Z. f. G. Nr. 7 a. S. 317. Reifferscheid, Zum Kampfe gegen die Fruchtabtreibung. Z. f. G. Nr. 9.

S. 518. Reschke, Zur Entstehung des Dekubitus. Med. Klin. Nr. 8. S. 238. Riolte, Diffuse

Hyperplasie der Dezidua und Biutungen am Ende der

Schwangerschaft. Gynéc. obstét. IX. Nr. 1. S. 23. Riehl, Anatomisch-klinische Studie über

Placenta marginata. Gynéc. 
obst, IX. 1. S. 82. Range, Die Gynäkologie des praktischen Arztes. Med. Klin. 7/218, 8/251, 9/285, 10/318. Schickele, 1. Studien über die Eierstockfunktionen. Es besteht keine Be-

ziehung zwischen Theka-Drüse und normaler oder unnormaler Menstruation. S. 1. - 2. Kann der gelbe Körper die Quelle neuer ausgiebiger

intraperitonealer Blutung sein ? S. 9. - 3. Wahre und falsche Tumoren

der Plazenta. S. 50. - 4. Schwangerschaftstoxämie des Geburtsschoks.

Plötzlicher Tod nach der Entbindung. Gynéc. Obsfcétr. IX. Nr. 1.

S. 123. Schnabel und Kasarnowsky, Trypaflavin als Slreptokokken-überempfindlich

machende Substanz. Klin. Woch. Nr. 9. S. 346. Schmitt, Über die Behandlung der

Dysmenorrhöe iind Sterilität. Z. f. G.

Nr. 7 a. S. 287.

$32 \hat{v}$

Persoaalien und Tagesnachrichten.

Sellheim, Uterusexstirpation oder Kastration? Munch, med. Woeh. Nr. 8.

S. 239. Smith, Schmerzen bei gynäkologischen Leiden. Surg. gyn. obst. Febr. 24.

S. 216. Steiner, Abdominoreopsie. Surg. gyn. obst. Febr. 24. S. 266. Stephansky, Über eine akute epidemische gangränöse Erkrankung der

äußeren Geschlechtsteile bei Kindern. Dtsch. med. Woch. Nr. 10.

S. 305. Streibel, Fall von Uterus dupl. bicorn. myomat. bei gleichzeitig bestehendem

Anus vestibularis. Z. f. G. Nr. 7. S. 262. Stabler, Zur Frage des sogen. Kleinschen Tumors und

über die Entstehung

des Oberflächenpapillomes. Z. f. G. Nr. 9. S. 532. Stuhl, Zur Behandlung der

Bauchfelltuberkulose mit Tuberkulín. Med.

Klin. Nr. 10. S. 312. Stukowski, Über Klitoriszysten. Munch, med. Woch. Nr. 8. S. 238. Stutzin, Über die Unfruchtbarmachung von Schädlingen. Med. Klin. Nr. 10.

S. 331. Taschek, Über zwei durch Operation geheilte Fälle von schweren Uterus-

verletzungen, verursacht durch Fruchtabtreibung. Z. f. G. Nr. 7 a.

S. 336. Vassallo, Die Wassermann-Reaktion in der Gynäkologie. La cl $1 \frac{1}{8} \mathrm{ca}$ obst.

Febr. 24. S. 46. Wagner-Hohenlobbese, Einfache und sichere Methode der Operation des typischen Schenkelbruches beim Weibe. Z. f. G. Nr. 7. S. 260. Weibel, W., Einführung in die gynäkologische Diagnostik. III. Aufl.

J. Springer, Berlin 1924.

Strahlentherapie.

Kok, Weitere tierexperimentelle Studien über die Wirkung der Röntgen-strahlen auf das

Karzinom. Dtsch. med. Woch. Nr. 10. S. 298.

Personalien und Tagesnachrichten.

Dr. Hans Runge, 1. Assistent der Universitäts-Frauenklinik in Kiel, hat sich als Privatdozent an der dortigen tlniversität niedergelassen.

Die Kölner Provinzial-Hebammen-Lehranstalt ist von der Rheinischen

Provinzialverwaltung an die Staclt Köln verpachtet worden. Die Aus-

und Fortbildungskurse für die Hebammen der Rheinprovinz werden bis

auf weiteres nur noch in Elberfeld abgehalten. Die Elberfelder Anstalt

wird erheblich vergrößert.

Die Niederrheinisch -Westfälische Gesellschaft für Gynäkologie und Geburtshilfe hat am 18. Mai zu Ehren ihres 25 jährigen Bestehens in Düsseldorf eine Festsitzung abgehalten. Dieselbe 
war schon vor Jahres-frist fällig, mußte damals aber wegen der Verkehrsschwierigkeiten infolge des Ruhreinbruches verschoben werden. 